\title{
A Novel Low Power CMOS VGA
}

\author{
Du Yongqian \\ Northwestern Polytechnical University, Xi’an, China
}

\author{
Liu Shibin, Li Guifang \\ Northwestern Polytechnical University, Xi’an, China
}

\begin{abstract}
A low-power Variable Gain Amplifier (VGA) is demonstrated in a commercial $0.18 \mu \mathrm{m}$ CMOS technology, with a chip size of $0.019 \mathrm{~mm}^{2}$. To compensate the threshold voltage process variation, a control voltage level shift (CVLS) methodology is proposed. The VGA was measured with a dynamic range of $29 \mathrm{~dB}$ with a $\pm 0.62 \mathrm{~dB}$ error. The total power consumption is $186 \mu \mathrm{W}$, and the bandwidth is $26 \mathrm{MHz}$.
\end{abstract}

Keywords-VGA; exponential current; low power; subthreshold; linear-in-dB

\section{INTRODUCTION}

Variable gain amplifier (VGA), the gain of which is a linear-in- $\mathrm{dB}$ function of control voltage or current, plays an important role in stabilizing the power of the receiver's output. Current trends make the power-efficiency requirement become an inevitable consideration. However, one great challenge is the design of low-power VGA. Unfortunately, the power of traditional PNP, pseudo-exponential approximation, digital controlled amplifier and some other solutions for VGA are too big for portable receivers ${ }^{[1][2][3][4]}$.

The sub-threshold methodology has been verified as a realistic low-power solution ${ }^{[5][6]}$. The well-proved exponential $\mathrm{I}-\mathrm{V}$ curvature of MOS transistor is the base for sub-threshold circuit design ${ }^{[5][6][7][8] \text {. }}$

$$
I_{\mathrm{ds}}=I_{\mathrm{s} 0} S \exp \left(\frac{V_{\mathrm{gs}}-V_{\mathrm{th}}-V_{\mathrm{off}}}{n V_{\mathrm{T}}}\right)\left(1-\exp \frac{-V_{\mathrm{ds}}}{V_{\mathrm{T}}}\right)
$$

Where: $S$ is the aspect ratio of the MOS transistor, $V_{\mathrm{ds}}$ is its drain and source voltage difference. $V_{\mathrm{T}}=k T / q\left(26 \mathrm{mV} @ 27^{\circ} \mathrm{C}\right)$. $n$ is a parameter defined as the differential of the gate voltage $V_{\mathrm{G}}$ to the cut off voltage $V_{\mathrm{p}}$, and it ranges from 1 to $2 . V_{\text {off }}$ is the gate-source voltage tested when $I_{\mathrm{ds}}=0$, which varies from different process, and for the current process, $V_{\text {off }}=130 \mathrm{mV} . I_{\text {so }}$ is parameter defined by process.

When $V_{\mathrm{ds}}>4 V_{\mathrm{T}}$, the last term of $I_{\mathrm{ds}}$ can be neglected, then the exponential I-V characteristic can be simplified as:

$$
I_{\exp }=I_{\mathrm{ds}}=I_{s 0} S \exp \left(\frac{V_{\mathrm{gs}}-V_{\mathrm{th}}-V_{\text {off }}}{n V_{\mathrm{T}}}\right)
$$

The above exponential I- $\mathrm{V}$ characteristic has been verified in low noise amplifier (LNA), voltage controlled oscillator (VCO) and voltage reference with satisfactory performance [5][6] [8]

As can be seen from Equation (2), $I_{\exp }$ shows a direct correlation with the threshold voltage $V_{\text {th }}$. However, as the threshold voltage may have great variation with the process. Thus, two main problems of the sub-threshold MOS transistor exsit: 1) MOS should be biased in sub-threshold region reliably; 2) the process variation of the threshold voltage $V_{\text {th }}$ may drive MOS transistor out of sub-threshold region. Both these two problems can collapse the performance and even the function of the exponential I-V curvature.

The purpose of this paper was to present a low power VGA, where, the above two problems are compensated by a CVLS methodology.

\section{CIRCUIT DESIGN}

Fig. 1 shows the configuration of the VGA. It is mainly composed of one stage of VGA amplifier cell (VGA-cell), which has two amplifiers in series, a V-I converter and a Vthdetector. The VGA-cell is the core circuit to realize the exponential voltage gain under the control of the exponential current $I_{\text {exp }}$, which is generated by the Sub-threshold transistor Mexp. To solve the problems of the sub-threshold transistor mentioned above, a CVLS scheme is introduced, which guarantees the Mexp always be biased in sub-threshold region and can simultaneously cancel the process variation. The summing resistor R0, V-I converter and the Vth-detector are the key circuits for CVLS, the theory of which will be demonstrated in the following text.

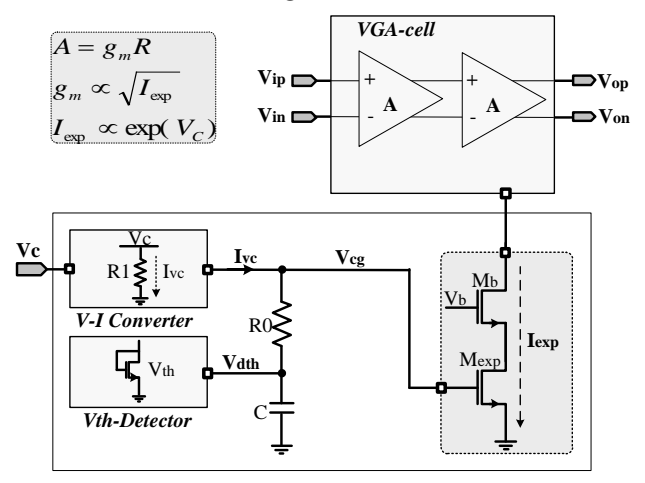

Fig.1. Architecture of the Proposed VGA

The schematic of the V-I converter is shown in Fig.2, and $V_{\text {cg }}$ can be deduced as:

$$
V_{c g}=V_{R 0}+V_{d t h}=\frac{M V_{C}}{N R_{1}} R_{0}+V_{d t h}
$$

The schematic of the Vth-detector is shown in Fig.3. Transistor M3 M6 are biased in sub-threshold region with an exponential I-V curvature. The negative feedback loops formed by Amp1 forces $V_{\mathrm{a} 1}=V_{\mathrm{a} 2}$. Thus: 


$$
V_{\text {dth }}=V_{\text {th }}+V_{\text {off }}+n V_{\mathrm{T}} \ln \left[\frac{n V_{\mathrm{T}}}{I_{\text {s0 }} R_{2} S_{3}} \ln \left(\frac{S_{5}}{S_{6}} \frac{S_{4}}{S_{3}}\right)\right]
$$

When $V_{\mathrm{cg}}=V_{\mathrm{gs}}$ is applied on Mexp, $I_{\exp }$ can be then expressed as:

$$
I_{\text {exp }}=I_{s 0} S_{M \exp } \exp \left\{\ln \left[\frac{n V_{\mathrm{T}}}{I_{s 0} R_{2} S_{3}} \ln \left(\frac{S_{5}}{S_{6}} \frac{S_{4}}{S_{3}}\right)\right]+\frac{M R_{0}}{N R_{1} n V_{\mathrm{T}}} V_{C}\right\}
$$

The first item in the brace of the above exponential function and the coefficient of $V_{\mathrm{c}}$ can be considered as constants, which are supposed to be $\alpha$ and $\beta$ respectively. Then:

$$
I_{\text {exp }}=I_{s 0} S_{M \exp } \exp \left(\alpha+\beta V_{C}\right)
$$

Which shows that $I_{\exp }$ is only decided by the control voltage $V_{\mathrm{c}}$, as $V_{\text {th }}$ variation is compensated by $V_{\text {dth }}$. Moreover, when all design parameters are reasonably set to make $\left(M V_{\mathrm{c}} R_{1}\right) /\left(N R_{2}\right)+n V_{\mathrm{T}} \alpha<\left|V_{\mathrm{off}}\right|, V_{\mathrm{cg}}<V_{\text {th }}$ always holds. Thus, with the help of $V_{\text {dth }}$ and CVLS scheme, transistor Mexp is always biased in sub-threshold region reliably even with large process variation. Similar deduction and simulation results related to above exponential I-V curvature have also been illustrated in the author's previous work ${ }^{[9]}$.

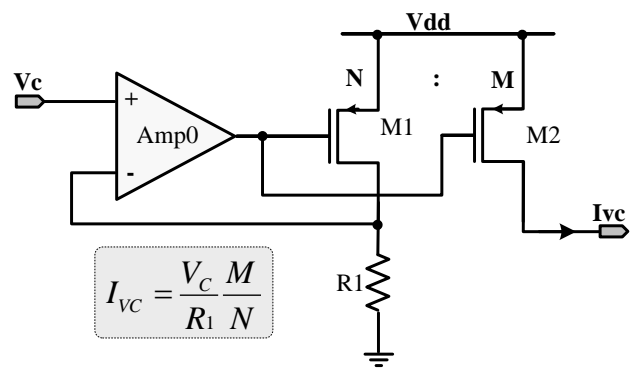

Fig.2. Circuit of V-I Converter

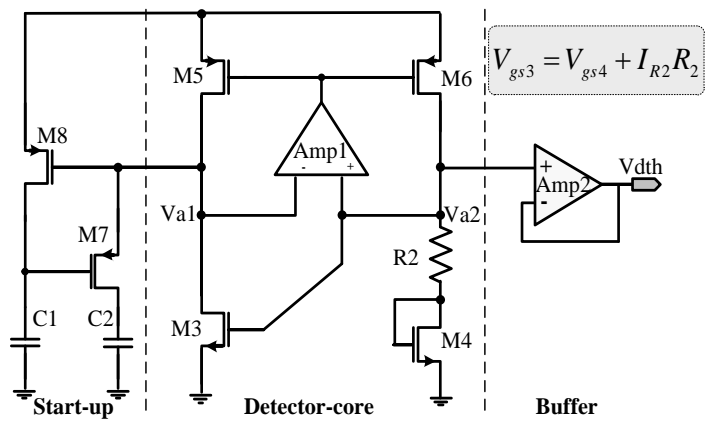

Fig.3. Schematic of Vdth-generator

The realization of the VGA-cell is shown in Fig.4, which is composed of two amplifiers in series. The trans-conductance of MOS transistor is: $g_{\mathrm{m}}=\left(u_{\mathrm{n}} c_{\mathrm{ox}} S I_{\mathrm{ds}}\right)^{1 / 2}$. When control current $I_{\text {exp }}$ is applied on the VGA-cell and based on Fig.1, the final gain of the VGA, $G_{\text {exp }}$, indicates an exponential gain of the VGA, which is shown as follows:

$$
\ln \left(G_{\text {exp }}\right)=\gamma+\alpha+\beta V_{C}
$$

Where $\gamma$ is a constant which can be expressed as:

$$
\gamma=\ln \left(R_{3} R_{4} \mu_{n} C_{o x} S_{9 \sim 12} \frac{m}{2} I_{50} S_{M \exp }\right)
$$

Based on Equation (7), it should be noted that: as $V_{\text {th }}$ variation of the exponential control current $I_{\exp }$ has been compensated by CVLS, the gain of the VGA-cell shows a linear correlation with the control voltage $V_{\text {c }}$. Finally, despite the great process variation of the MOS transistor, the proposed VGA has a good linearity.

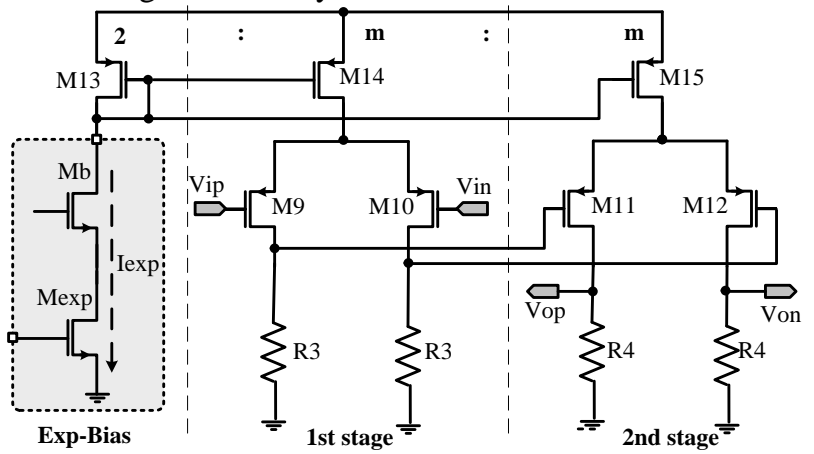

Fig.4. Schematic of VGA amplifier cell

\section{MEASUREMENT ResultS}

The proposed VGA was fabricated in a $0.18 \mu \mathrm{m}$ CMOS technology. Fig.5 shows the die photo of the proposed VGA with a die area of $0.019 \mathrm{~mm}^{2}$.

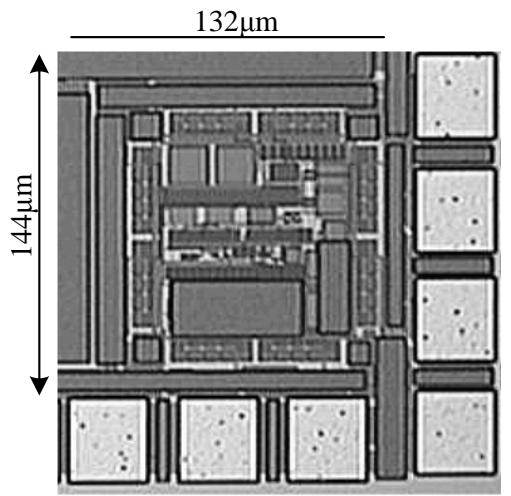

Fig.5. Die photo of the proposed circuits with test pads

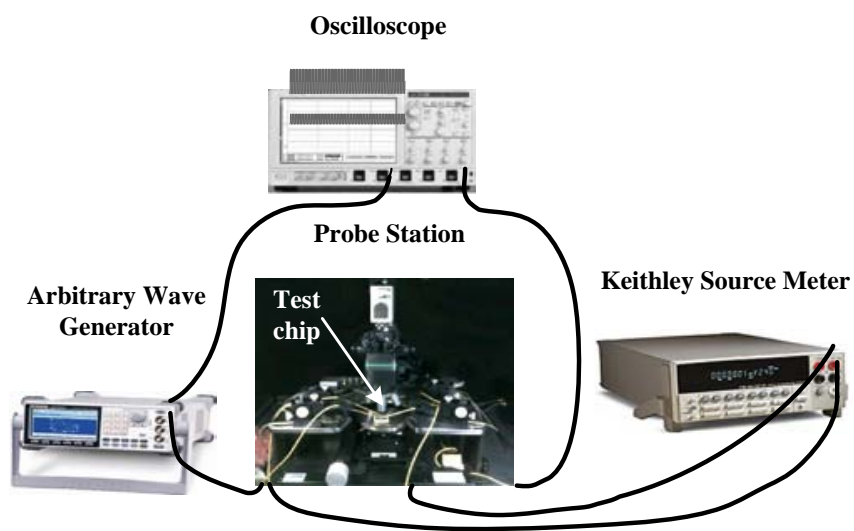

Fig.6. Diagram of the test setup

The performance measurements of the proposed VGA are made on-wafer with the help of a probe station. The diagram of the complete test setup is shown in Fig.6, where the 
sinusoidal input is generated by an arbitrary wave generator. Moreover, a Keithley source meter is used as the power source of the VGA, which can simultaneously be used to detect the current consumption of the VGA.

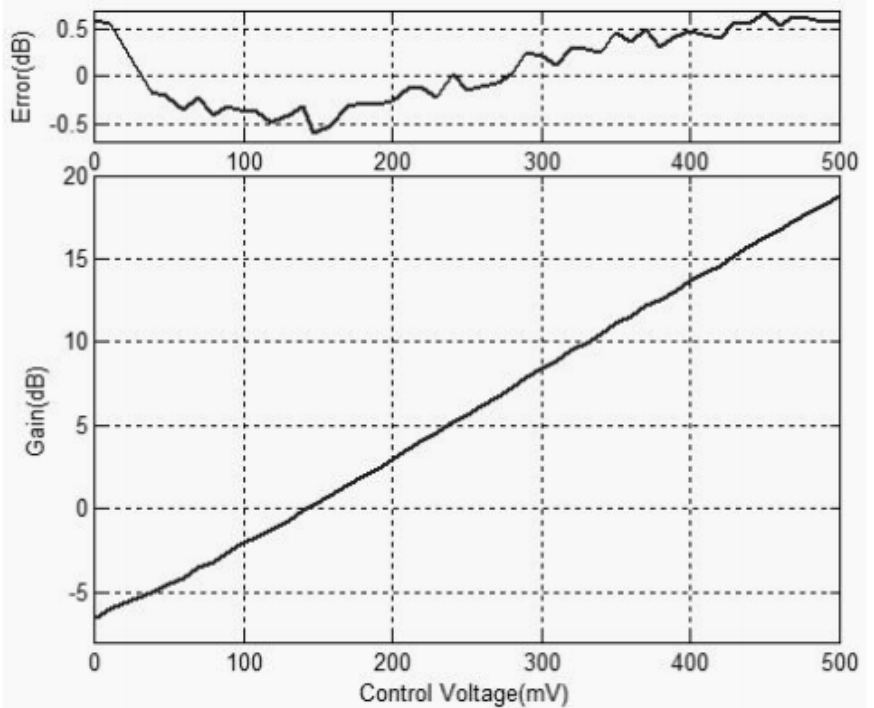

Fig.7. Gain and $\mathrm{dB}$ error with respect to $V_{\mathrm{c}}$

The measured gain of the proposed VGA is shown in Fig.7. As can be seen, with the control voltage $V_{\mathrm{c}}$ ranging from 0 to $500 \mathrm{mV}$, the dynamic decibel range is up to $29 \mathrm{~dB}$, and the $\mathrm{dB}$ error is less than $\pm 0.62 \mathrm{~dB}$.

Table 1 shows the performance of the proposed VGA compared with previously reported VGAs. The total power consumption is $186 \mu \mathrm{W}$, which is tested by the Keithley source meter.

TABLE I. Performance summary of the Proposed VGA

\begin{tabular}{|l|c|c|c|c|}
\hline Parameters & $\begin{array}{c}\text { This } \\
\text { Work }\end{array}$ & {$[1]$} & {$[2]$} & {$[4]$} \\
\hline Power source/V & 1.8 & 1.8 & 1.8 & 1.8 \\
\hline Number of stages & 1 & 1 & 3 & 3 \\
\hline Gain range/dB & 29 & 48 & 60 & 95 \\
\hline $\mathrm{dB}$ error/dB & \pm 0.62 & NA & NA & NA \\
\hline Bandwidth/MHz & 26 & 3 & 16 & 32 \\
\hline Power/mW & 0.186 & 0.55 & 3.6 & 6.5 \\
\hline
\end{tabular}

\section{CONCLUSION}

A low power VGA is demonstrated with a power of $186 \mu \mathrm{W}$, where the CVLS scheme compensates the $V_{\text {th }}$ variation and safely biases MOS in sub-threshold region. As a result, the VGA shows good linearity even under big process variation. The measured dynamic range is $29 \mathrm{~dB}$, and the $\mathrm{dB}$-error is less than $\pm 0.62 \mathrm{~dB}$. The bandwidth is $26 \mathrm{MHz}$, which is big enough for Zero-IF receiver.

\section{ACKNOWLEDGMENT}

This project is supported in part by the Fundamental Research Funds for the Central Universities G2015KY0105.

\section{REFERENCES}

[1] Shang Hisen Yang, etc, A 48-dB dynamic gain range/stage linear-in-dB low power variable gain amplifier for direction conversion receivers, IEEE J. of Solid-State Circuits, vol. 38, November, 2011, pp. 1602-1608.

[2] Q. Q. Lei, etc, A Low-power CMOS VGA with 60-dB linearly controlled gain range for GPS application, 2008 IEEE Solid-State and Integrated-Circuit Technology, October 2008, pp. 1669-1675.

[3] D. G. Liang, Q. Ye, A 96-dB CMOS programmable gain amplifier for Low-IF receiver, 2008 IEEE Communication, Circuits and Systems, pp. 101104, May 2008.

[4] Q.-h Duong, etc, A 95-dB linear low-power variable gain amplifier, IEEE Tans. Circuits Syst. I. vol. 53, pp. 1648-1657, Aug. 2006.

[5] Hanil Lee, etc, A Subthreshold Low Phase Noise CMOS LC VCO for Ultra Low Power Applications, IEEE Microwave and Wireless Components Letters, Vol. 17, No. 11, Nov. 2007.

[6] H. Lee and S. Mohammadi, A $3 \mathrm{GHz}$ subthreshold CMOS low noise amplifier, in Proc. IEEE RFIC Symp., Jun. 2006, pp. 494-497.

[7] Weidong Liu, etc, BSIM3v3.2.2 MOSFET Model Users' Manual, Department of Electrical Engineering and Computer Sciences University of California, Berkeley, CA 94720.

[8] Yongqian Du, et al., A reliable demodulator and a power regulation circuit with an accurate and small-size voltage reference in UHF RFID, Microelectron. J. 44 (2012), pp. 72-79.

[9] Du Yongqian, Zhuang Yiqi, Li Xiaoming, and Liu Weifeng, New Type of Low-Power Exponential Current Generator for VGA. Journal of University of Electronic Science and Technology of China. Vol.43, No.2, Mar. 2014, pp. 282-286. 